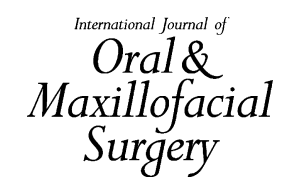

Clinical Paper

Trauma

\section{Impact of trauma and surgical treatment on the quality of life of patients with facial fractures}

\author{
J.J. Conforte, C.P. Alves, M.delP.R. Sánchez, D. Ponzoni: Impact of trauma and \\ surgical treatment on the quality of life of patients with facial fractures. Int. J. Oral \\ Maxillofac. Surg. 2016; 45: 575-581. (C) 2015 International Association of Oral and \\ Maxillofacial Surgeons. Published by Elsevier Ltd. All rights reserved.
}

\begin{abstract}
This study assessed the impact of oral and maxillofacial trauma and surgical treatment on the quality of life of patients. The study included 66 patients (age range 18-65 years) with facial fractures; 33 required surgical treatment and 33 required conservative (non-surgical) treatment. Quality of life was evaluated by applying the Oral Health Impact Profile questionnaire (OHIP-14) immediately after diagnosis of the trauma (T1), 30 days after surgery or trauma (T2), and 90 days after surgery or trauma (T3). For the control group (conservative treatment), there was a change in quality of life at T1 and T2. A change in quality of life was found for all of the surgical patients, regardless of the type of fracture and the observation period analyzed. There was no statistical difference when T1, T2, and T3 were compared in cases of zygomatic, Le Fort I, and nasal fractures, however there was an improvement in the quality of life of patients with mandibular fractures $(P=0.0102)$ and multiple facial fractures $(P=0.0097)$ at T3. Facial trauma caused the greatest impact on the quality of life of surgical patients at T1. The surgical treatment significantly improved quality of life for patients with mandibular and multiple facial fractures.
\end{abstract}

\author{
J. J. Conforte ${ }^{1}$, C. P. Alves ${ }^{1}$, \\ M. delP. R.Sánchez ${ }^{1}$, D. Ponzoni ${ }^{2}$ \\ ${ }^{1}$ Araçatuba School of Dentistry - UNESP, São \\ Paulo, Brazil; ${ }^{2}$ Department of Surgery and \\ Integrated Clinic, Araçatuba School of \\ Dentistry - UNESP, São Paulo, Brazil
}

Key words: facial injuries; quality of life; facial bones; maxillary fractures; face.

Accepted for publication 26 November 2015 Available online 24 December 2015
Facial trauma is considered one of the most devastating events in a patient's life as it usually results in physiognomic deformities and possible emotional consequences. $^{1-5}$ Due to the increase in occurrence of facial trauma during the last four decades, important discussions on this topic have taken place. Currently, it is thought that the main aetiological factors are alcohol and drug use, car accidents, and increasing urban violence. However, the occurrence of these factors varies greatly according to the region studied. ${ }^{6-13}$

Oral and maxillofacial injuries can occur in isolation or be part of a larger trauma. For this reason, multidisciplinary examination involving specialties such as ophthalmology, plastic surgery, maxillofacial surgery, and neurosurgery is extremely important when making a neurological assessment.

Several studies on the impact of oral health problems on quality of life have been reported. ${ }^{5,14-16}$ However, few studies have investigated this issue in patients with facial trauma. Quality of life is currently considered an important marker for assessment in oral health studies. ${ }^{15,16}$ This has led to the development of new instruments to assess quality of life. ${ }^{17}$

The Oral Health Impact Profile (OHIP) questionnaire is one of the most commonly used instruments; it has been used in various studies across different cultures 
and socio-demographic profiles. The OHIP was developed in order to provide a comprehensive measurement of the dysfunction, discomfort, and disability associated with oral conditions as reported by the individual. ${ }^{18,19}$ OHIP analyzes the different dimensions of functional patterns. These dimensions are functional limitation (e.g., difficulty chewing), pain (e.g., sensitivity of teeth), psychological discomfort (e.g., personal embarrassment), physical disability (e.g., changes in diet), psychological disability (e.g., reduced concentration), social disability (e.g., avoiding social contact), and incapacitation (e.g., being unable to work productively). ${ }^{19,20}$

In 1997, Slade described an abridged version of the OHIP, called OHIP-14, which was derived from the original version, OHIP-49. ${ }^{18}$ In this version, the author maintained the dimensional concepts of health of the original questionnaire. The author suggested that this new instrument might be useful to quantify the level of impact on the quality of life of patients. Among the 14 questions of OHIP-14, 10 relate to the psychological and behavioural impact and four address each of the remaining general dimensions. Therefore, OHIP-14 can be considered one of the best detectors of the psychosocial impact in a population.

The objective of this study was to assess the impact of oral and maxillofacial trauma and surgical treatment on the quality of life of patients attending a centre for surgery and oral and maxillofacial traumatology in Araçatuba, Brazil.

\section{Methods}

The participants were patients with facial trauma undergoing treatment at a centre for surgery and oral and maxillofacial traumatology in Araçatuba, Brazil, from August 2013 to July 2014. The project was approved by the ethics committee of the dental school.

The patients included in this study were adults suffering from facial bone fractures. Some of these patients required surgical treatment, while others were indicated for conservative treatment. All were followed-up for 90 days. Patients were excluded if they had mental disabilities, had neurological sequelae, were unable to respond appropriately to the examination questions and the questionnaire, or missed one of the follow-up consultations within the 90 days.

A validated version of the OHIP-14 questionnaire (Oral Health Impact Profile-short form) was applied to participants in order to evaluate their quality
Table 1. Brazilian version of the OHIP-14 questionnaire.

\begin{tabular}{|c|c|}
\hline $\begin{array}{l}\text { Question } \\
\text { After suffering from trauma to the face? } \\
\text { After surgery? }\end{array}$ & $\begin{array}{c}\text { Reply } \\
0=\text { never } \\
1=\text { rarely } \\
2=\text { sometimes } \\
3=\text { repeatedly } \\
4=\text { always }\end{array}$ \\
\hline 1. You have had problems saying some words... & 01234 \\
\hline 2. The taste of foods has worsened... & 01234 \\
\hline 3. You have felt strong pain in your mouth... & 01234 \\
\hline 4. You have felt uncomfortable eating any food... & 01234 \\
\hline 5. You have felt uncomfortable... & 01234 \\
\hline 6. You have felt stressed... & 01234 \\
\hline 7. Your diet has been hampered... & 01234 \\
\hline 8. You had to stop taking your meals... & 01234 \\
\hline 9. You have found it hard to relax... & 01234 \\
\hline 10. You have already felt a bit embarrassed... & 01234 \\
\hline 11. You have felt irritated by other people... & 01234 \\
\hline 12. You have found it difficult to carry out your daily activities... & 01234 \\
\hline 13. You have felt that life in general has worsened... & 01234 \\
\hline 14. You have not been able to carry out your daily activities... & 01234 \\
\hline
\end{tabular}

of life. ${ }^{21}$ Interviews were carried out by a single interviewer, previously trained in the application of the questionnaire. ${ }^{21}$ The original OHIP-14 questions underwent minor adaptation: the words "their teeth and dentures" were replaced with the words "after suffering facial trauma", and "after surgical treatment".

The OHIP-14 was applied three times during the 90-day follow-up: immediately after diagnosis of the trauma (T1), 30 days after the day of surgery or indication for conservative treatment (T2), and 90 days after surgery or the indication for conservative treatment (T3). During follow-up interviews, the patients reported their problems as perceived by themselves, and the problems were classified according to their frequency.

Table 1 lists the OHIP-14 questions used in this study. The possible response to each question was 'never', 'rarely', 'sometimes', 'repeatedly', or 'always'; these were scored as $0,1,2,3$, and 4 , respectively. The total score for the OHIP14 ranges from 0 to 56 .

The questions assessed the following problems: pronunciation (question 1), taste (question 2), pain (question 3), discomfort when eating (question 4), oral discomfort (question 5), nervous tension (question 6), hampered eating (question 7), interruption of meals (question 8), difficulty relaxing (question 9), embarrassment (question 10), irritation with other people (question 11), difficulty in carrying out daily activities (question 12), unsatisfactory life (question 13), and functional incapacity (question 14). These questions were grouped into seven domains (two questions per domain), as shown in Table 2 .
The score for each item for each individual was added up and the final score obtained (score range 0-56). The mean OHIP-14 score was calculated for each type of fracture at each observation point. The Shapiro-Wilk normality test was performed. The scores for the three observation periods were then compared statistically using the Kruskal-Wallis non-parametric test. In the case of a significant difference, the Dunn test was applied. The significance level was set at 5\%. The control group (conservative treatment) was compared to the treated group (surgical treatment).

\section{Results}

From August 2013 to July 2014, 398 patients with facial trauma were treated at the centre for oral and maxillofacial surgery and traumatology. Of these 398 patients, 125 were diagnosed with facial fractures, 53 of whom required surgical treatment and 72 of whom were treated conservatively. The study included 66 patients (47 male and 19 female), aged between 18 and 65 years, who met the established criteria.

Table 2. Domains of the OHIP questionnaire according to the problems presented.

\begin{tabular}{lc}
\hline Domain & Questions \\
\hline Functional limitation & $1-2$ \\
Physical pain & $3-4$ \\
Psychological discomfort & $5-6$ \\
Physical incapacity & $7-8$ \\
Psychological incapacity & $9-10$ \\
Social incapacity & $11-12$ \\
Social disadvantage & $13-14$ \\
\hline
\end{tabular}

OHIP, Oral Health Impact Profile. 


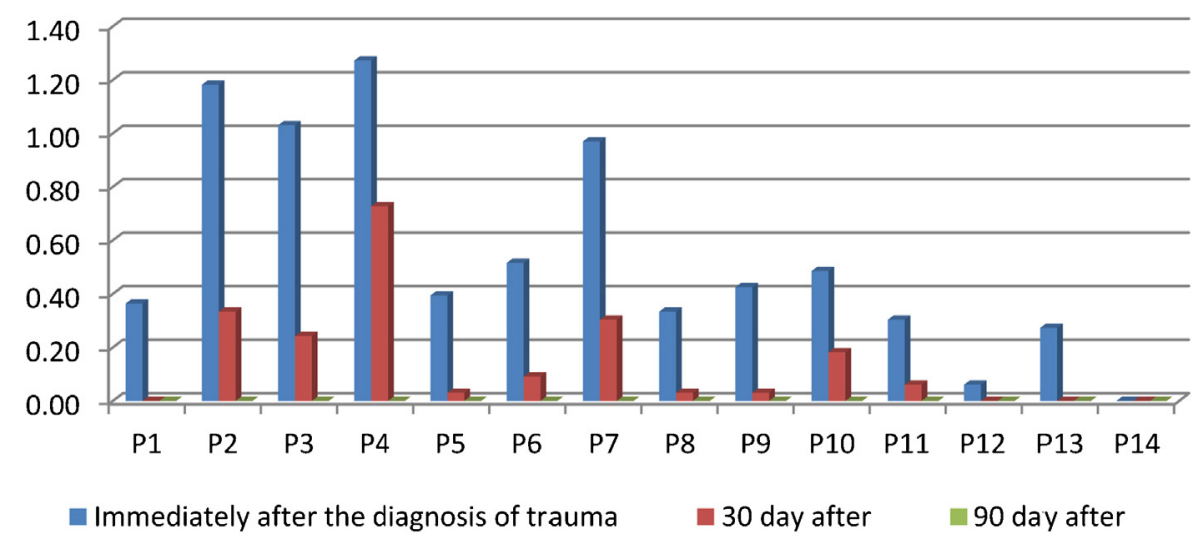

Fig. 1. Patients indicated for conservative treatment. Distribution of OHIP-14 domains for the three evaluation periods. (Q1: problem in saying a word; Q2: the taste of food has worsened; Q3: has felt strong pain in the mouth; Q4: has felt uncomfortable when eating certain food; Q5: has felt uncomfortable; Q6: has felt stressed; Q7: their diet has been hampered; Q8: has had to stop their meals; Q9: has struggled to relax; Q10: has felt a bit embarrassed; Q11: has been irritated by other people; Q12: has found it difficult to carry out daily activities; Q13: has felt that life in general has become worse; Q14: has been unable to perform their daily activities.)

The control group consisted of 33 patients with facial fractures who underwent conservative treatment; nine of them had fractures of the zygomatic complex, nine had a mandibular fracture, and 15 had nasal bone fractures. The treatment group included 33 patients with various types of facial fracture: mandibular fracture ( $n=12$ patients), zygomatic complex fracture $(n=9)$, multiple fractures of the facial bones $(n=7)$, nasal fracture $(n=3)$, and Le Fort type I fracture $(n=2)$.

The results obtained from the questionnaire at times $\mathrm{T} 1, \mathrm{~T} 2$, and $\mathrm{T} 3$ are presented in Figs 1 and 2 and in Tables 3 and 4.

In the treatment group, facial fractures altered the quality of life for all patients, regardless of the type of fracture and the time period analyzed, as shown in Table 4 and Fig. 2. For the control group, there was an alteration in the quality of life at $\mathrm{T} 1$ and T2. At T3, not all the control group patients scored the questionnaires, showing that there was no change in quality of life during this period.

\section{Statistical analysis}

The Shapiro-Wilk normality test was carried out for the data regarding each type of fracture at the respective observation periods (T1, T2, and T3). The non-parametric Kruskal-Wallis test was then used for comparisons among the observation periods. For the treatment group, there was no statistical difference in quality of life when comparing T1, T2, and T3 in cases of zygomatic, Le Fort I, or nasal fractures. For mandibular fractures $(P=0.0102)$ and multiple facial fractures $(P=0.0097)$ there was a statistical difference among the observation periods, with an improvement in the quality of life at T3 (Table 5).

Comparing the control and treatment groups, there was a statistical difference for the three observation periods, with better quality of life in those patients who did not receive surgical treatment (Table 6).

\section{Discussion}

During the study period, 398 patients with facial trauma were treated at the centre for oral and maxillofacial surgery and traumatology in Araçatuba. At this centre, a medical team refers this type of patient to an emergency care unit with an oral and maxillofacial surgery team. Of these 398 patients, 125 presented with facial fractures. The remaining 273 presented with oedema and facial bruising with suspected

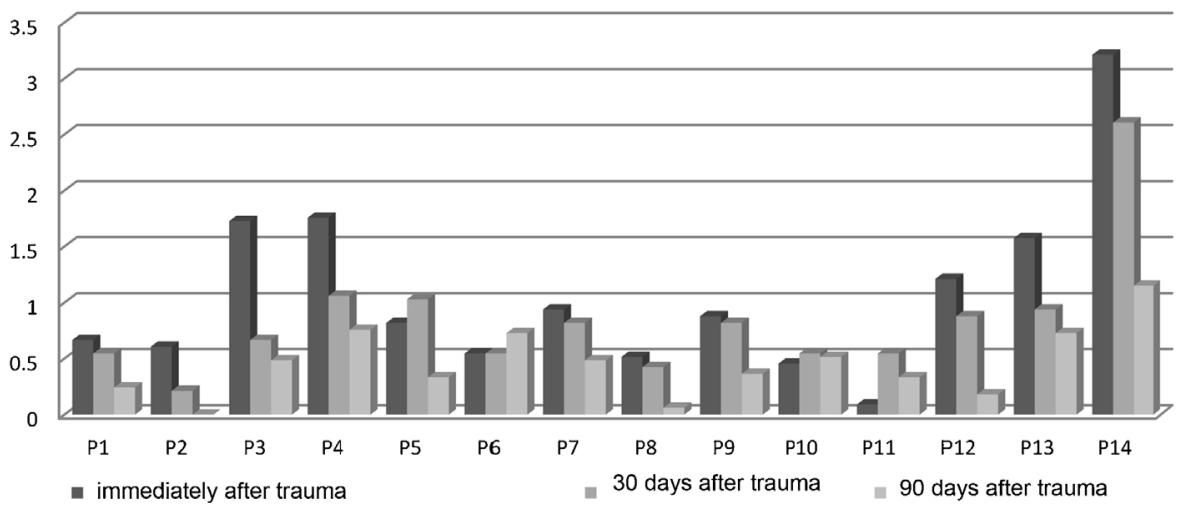

Fig. 2. Patients requiring surgical treatment. Distribution of OHIP-14 domains for the three evaluation periods. (Q1: problem in saying a word; Q2: the taste of food has worsened; Q3: has felt strong pain in the mouth; Q4: has felt uncomfortable when eating certain food; Q5: has felt uncomfortable; Q6: has felt stressed; Q7: their diet has been hampered; Q8: has had to stop their meals; Q9: has struggled to relax; Q10: has felt a bit embarrassed; Q11: has been irritated by other people; Q12: has found it difficult to carry out daily activities; Q13: has felt that life in general has become worse; Q14: has been unable to perform their daily activities.) 


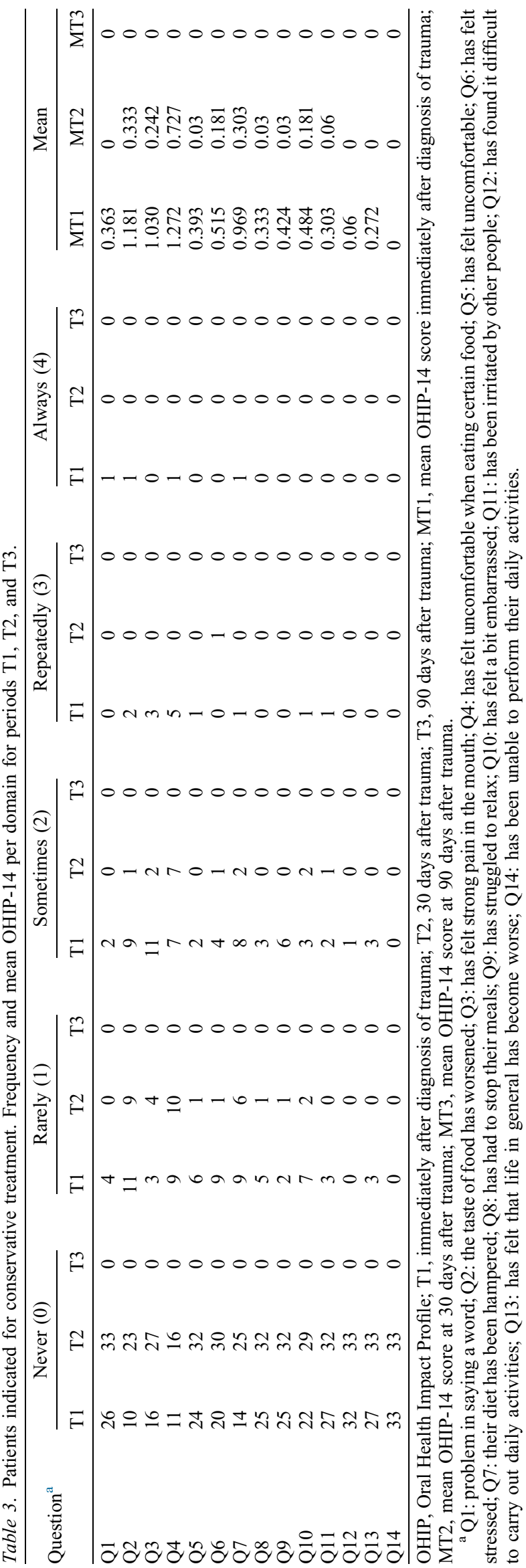

fractures, soft tissue injuries, and dentoalveolar trauma.

The control group in this study consisted of patients for whom conservative treatment was indicated. They were suffering from zygomatic bone fractures without displacement (nine patients), mandibular condyle fractures (nine patients), and nasal fractures with preservation of the nasal pyramid and no obstruction of the airspace (15 patients).

Not all of the 125 patients with facial fractures could be included in the study; patients who were not included were those who did not meet the inclusion criteria or did not attend the outpatient follow-ups during the study period.

Traumas generally modify the physical and psychiatric well-being of the patient in unexpected ways. For facial trauma, the biggest decline in quality of life is mainly immediately after the trauma. This was observed in the majority of the interviews in this study. Question 14 (functional incapacity) had the highest score, regardless of the type of fracture. This was because the patients evaluated had suffered the trauma during a productive phase of their lives, i.e., while still of working age. In addition, patients in the treated group required reduction and fixation surgery and were hospitalized while waiting for surgery.

During this stage immediately after the diagnosis of the trauma, patients often report limitations such as difficulty in eating (inability to eat in the way they did before the trauma, i.e., they can only eat liquid and pasty foods), difficulty pronouncing words, and pain (especially during eating). These complaints are related to the presence of oedema, lacerations of the soft tissue, trismus, limitations in mouth opening, and fractures that destabilize occlusion and the masticatory system. These conditions are frequent, especially in patients with multiple facial fractures. $^{22,23}$ These complaints are also reported in patients with mandibular fractures (the most frequent trauma in the sample). Mandibular fractures lead to mobility of the bone stumps, periosteum friction, and pain when moving. Patients who present Le Fort I type maxillary fractures report changes in their perception of taste, because they can taste blood. A unanimous complaint among patients is paresthesia in the affected region and the sensation of electric shock. ${ }^{24}$

Changes due to psychological discomfort are rarely reported considering that during this stage patients are hospitalized and under the care of a multidisciplinary team. Hospitalization restricts them from 


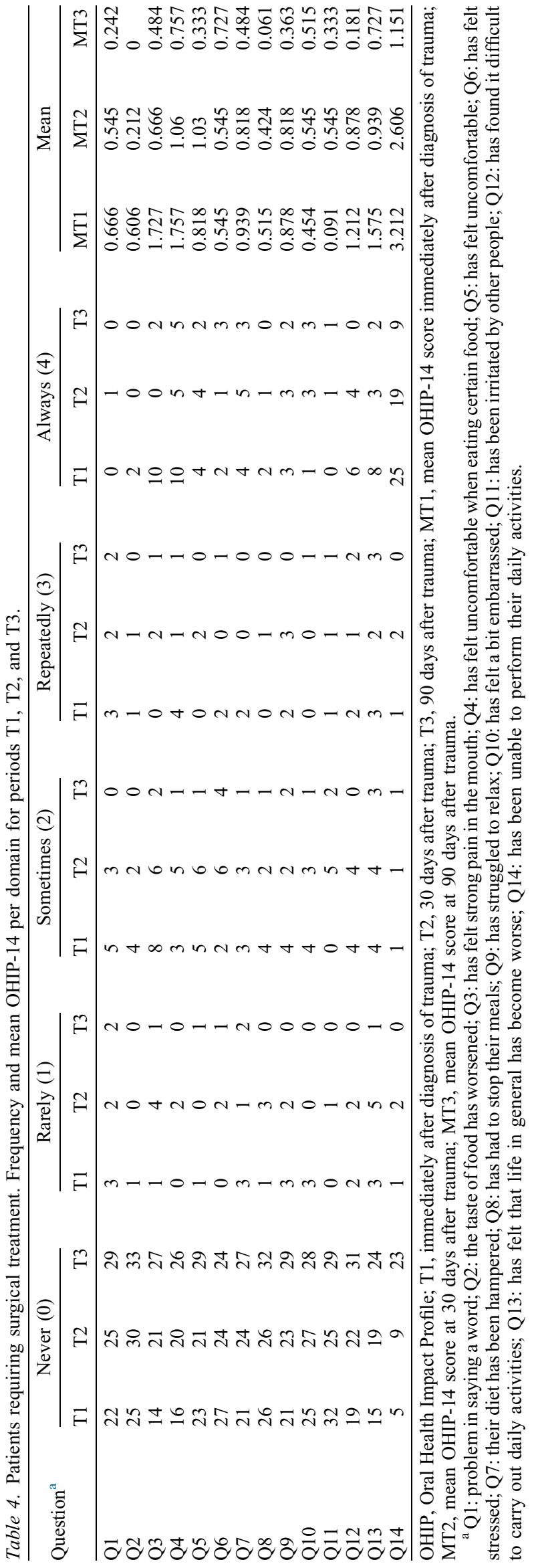

social contact and shields them from the embarrassment that they might feel regarding alterations in their appearance and/or questions from other people.

A direct relationship between the intensity of the trauma and the questionnaire scores was found. Patients who suffered multiple facial trauma and mandibular fractures had greater changes in their quality of life during this period.

In both groups, patients still reported an alteration in their quality of life at 30 days after the completion of surgery or sustaining the trauma. However, the alteration was lower than immediately after the trauma.

The social disadvantage domain and the feeling of discomfort when eating remained the most commonly reported factors by patients. Discomfort when eating is associated with the postoperative period or the conservative treatment that the patients are receiving during this period. They consume a liquid and soft diet and present trismus, which limits their ability to open the mouth. However, the pain is not as important, especially compared to the first observation period for mandibular fractures. ${ }^{22}$

The psychological domain is more often affected because patients feel uncomfortable. During this period (30 days after the trauma or surgery), patients have been discharged from the hospital and have resumed their social activities with family and friends, which makes them vulnerable to questions and comments about the changes caused by the trauma, such as scars, loss of teeth, and acquired deformities. $^{25}$ This may result in an increased risk of developing psychosocial sequelae. ${ }^{2-4}$

At 90 days after surgery, there was a significant improvement in the quality of life of patients who had received surgical treatment (especially mandibular fracture and multiple facial fracture patients). The reporting of pain decreased considerably, because there was a reduction in oedema, reduction of trismus, and the soft tissue had healed. There was no alteration in the quality of life among patients who had undergone conservative treatment during this observation period.

The report of changes in daily activities was most frequent in the surgical group, except when compared with the initial period. This is due to the fact that the majority of the patients were at a productive age (able to work) before the occurrence of the trauma.

For this observation period (90 days after the trauma or surgery), functional 
Table 5. Surgical treatment: statistical analysis using the Kruskal-Wallis test.

\begin{tabular}{llllll}
\hline & Zygomatic & Le Fort I & Nasal & Mandibular & Multiple \\
\hline $\mathrm{H}$ & 17.733 & 0.4101 & 50.863 & 91.653 & 92.752 \\
Degrees of freedom & 2 & 2 & 2 & 2 & 2 \\
$P$-value & 0.4120 & 0.8146 & 0.0786 & 0.0102 & 0.0097 \\
\hline
\end{tabular}

limitation is rarely reported or is nonexistent. The perception of taste returns to normal. Patients who cite problems in speaking report the reason as being tooth loss. Some patients report difficulties with hard consistency foods such as fruits and red meats, especially those who have suffered multiple fractures or mandibular fractures.

Overall, there was a reduction in the scores of the domains for the three observation periods. At T3, the score was zero for the conservative treatment group. Only the psychological domain increased at T2, when the patients were discharged from hospital and resumed their family and social lives. When comparing the two groups at the three observation points, the conservative treatment group had a significantly better quality of life for all three periods. This may have been due to the lack of displacement of fractures and exclusion of surgical treatment. It must be noted that the types of fracture included in this group were of lower intensity than in the surgical group. For example, a large number of patients in this group (15 patients) presented with only nasal fractures.

Surgical treatment, when indicated, appears to be a factor that contributes to improving the quality of life of these patients. Nevertheless, at 90 days after surgery, the patients still presented difficulties and had not resumed their daily activities normally. Further studies are needed to monitor these patients in the long term and establish strategies that may improve their quality of life.

Facial trauma had an impact on the quality of life of the patients analyzed. The greatest impact occurred immediately after diagnosis of the trauma. Surgical treatment improved the quality of life significantly in patients with mandibular

Table 6. Comparison between the treatment group and the control group: statistical analysis using the Tukey test.

\begin{tabular}{llll}
\hline & \multicolumn{1}{c}{ T1 } & \multicolumn{1}{c}{ T2 } & \multicolumn{1}{c}{ T3 } \\
\hline SD & 0.674 & 0.542 & 0.316 \\
Mean & 0.807 & 0.491 & 0.227 \\
$P$-value & 0.0354 & 0.0002 & 0.0001
\end{tabular}

$\mathrm{SD}$, standard deviation. and multiple facial fractures. The greater the intensity of the trauma, the greater the impact on the patient's quality of life.

\section{Funding}

None.

\section{Competing interests}

None.

This project was approved by the Ethics Committee of Araçatuba School of Dentistry - UNESP, São Paulo, Brazil (CAAE number 21344613.2.0000.5420).

\section{Patient consent}

Not required.

\section{References}

1. Sastry SM, Sastry CM, Paul BK, Bain L, Champion HR. Leading causes of facial trauma in the major trauma outcome study. Plast Reconstr Surg 1995;95:196-7.

2. Glynn SM, Shetty V. The long term psychological sequelae of orofacial injury. Oral Maxillofac Surg Clin North Am 2010;22:217-24. http://dx.doi.org/10.1016/ i.coms.2010.01.008.

3. Auerbach SM, Laskin DM, Kiesler DJ, Wilson M, Rajab B, Campbell TA. Psychological factors associated with response to maxillofacial injury and its treatment. J Oral Maxillofac Surg 2008;66:755-61.

4. Glynn SM. The psychosocial characteristics and need of patients presenting with orofacial injury. Oral Maxillofac Surg Clin North Am 2010;22:209-15. http://dx.doi.org/ 10.1016/j.coms.2010.01.003.

5. Bisson JI, Shepherd JP, Dhutia M. Psychological sequelae of facial trauma. $J$ Trauma 1997;43:496-500. http://dx.doi.org/ 10.1097/00005373-199709000-00018.

6. McDade AM, McNicol RD, Ward-Booth P, Chesworth J, Moos KF. The aetiology of maxillofacial injuries, with especial reference to the abuse of alcohol. Int J Oral Maxillofac Surg 1982;11:152-5.

7. Mackenzie EJ. Epidemiology of injuries: current trends and future challenges. Epide-

\section{Ethical approval} miol Rev 2000;22:112-9.
8. Wulkan M, Parreira Jr JG, Botter DA. Epidemiologia do trauma facial. Rev Assoc Med Bras 2005;51:290-5.

9. Chandra Shekar BR, Reddy C. A five-year retrospective statistical analysis of maxillofacial injuries in patients admitted and treated at two hospitals of Mysore city. Indian J Dent Res 2008;19:304-8.

10. Macedo JL, Camargo LM, Almeida PF, Rosa SC. Perfil epidemiológico do trauma de face dos pacientes atendidos no pronto socorro de um hospital público. Rev Col Bras Cir [Internet] 2008;35:9-13.

11. Martini MZ, Takahashi A, Oliveira Neto HG, Carvalho Júnior JP, Curcio R, Shinohara EH. Epidemiology of mandibular fractures treated in a Brazilian level I trauma public hospital in the city of São Paulo, Brazil. Braz Dent J 2006;17:243-8.

12. Montovani JC, Campos PL, Gomes MA, Moraes VR, Ferreira FD, Nogueira EA. Etiologia e incidência das fraturas faciais em adultos e crianças: experiência em 513 casos. Rev Bras Otorrinolaringol 2006;72: 235-41.

13. Slade GD, Spencer AJ, Locker D, Hunt RJ, Strauss RP, Beck JD. Variations in the social impact of oral conditions among older adults in South Australia, Ontario, and North Carolina. J Dent Res 1996;75: 439-50.

14. Krug EG, Sharma GK, Lozano R. The global burden of injuries. Am J Public Health 2000;90:523-6. http://dx.doi.org/10.2105/ AJPH.90.4.523.

15. Leão AT, Sheiram A. The development of measures of dental impacts on daily living. Community Dent Health 1996;13:22-6.

16. Adulyanon S, Vourapukjam J, Sheiham A. Oral impacts affecting daily performance in a low dental disease Thai population. Community Dent Oral Epidemiol 1996;24: 385-9.

17. Cohen LK, Jago JD. Toward the formulation of social dental indicators. Int J Health Serv 1976;6:681-98.

18. Slade GD. Derivation and validation of a short form oral health impact profile. Community Dent Oral Epidemiol 1997;25: 284-90.

19. Slade GD, Spencer AJ. Development and evaluation of the oral health impact profile. Community Dent Health 1994;11: $3-11$.

20. Allen PF, Locker D. Do item weights matter? An assessment using the oral impact profile. Community Dent Health 1997;14: 133-8.

21. Robson FC, Pordeus IA, Vale MP, Paiva SM. Validação do Oral Health Impact Profile-14. Pesqui Odontol Bras 2003;17: 176.

22. Tabrizi R, Bahramnejad E, Mohaghegh M, Alipour S. Is the frequency of temporomandibular dysfunction different in various mandibular fractures. J Oral Maxillofac Surg 2014;72:755-61. 
23. Boffano P, Roccia F, Gallesio C, Karagozoglu KH, Forouzanfar T. Infraorbital nerve posttraumatic deficit and displaced zygomatic fractures: a double-center study. J Craniofac Surg 2013;24:2044-6.

24. Markiewicz MR, Gelesko S, Bell RB. Zygoma reconstruction. Oral Maxillofac Surg Clin North Am 2013;25:167-201.
25. Patel PB, Stanton DC, Granquist EJ. Common dental and orofacial trauma evaluation and management. Med Clin North Am 2014;98:1261-79.

\section{Address:}

Daniela PonzoniQ2

Departamento de Cirurgia e Clínica Integrada Faculdade de Odontologia de
Araçatuba - UNESP

Rua José Bonifácio

1193

CEP 16015-050 São Paulo

Brazil

Tel: +55 183636 3270/18 99135 6561;

Fax: +55 1836363270

E-mail:dponzoni@foa.unesp.br 